\title{
IMECE2005-80396
}

\section{APPLICATION OF MONTE CARLO SIMULATIONS TO HYDRATED NAFION STIFFNESS PREDICTIONS}

\author{
Lisa Mauck Weiland* \\ Department of Mechanical Engineering \\ University of Pittsburgh \\ Pittsburgh, Pennsylvania 15261 \\ Email: Iweiland@engr.pitt.edu
}

\author{
Ralph C. Smith \\ Center for Research in Scientific Computation \\ Department of Mathematics \\ North Carolina State University \\ Raleigh, North Carolina, 27695 \\ rsmith@eos.ncsu.edu
}

\author{
Emily K. Lada \\ Statistical and Applied Mathematical Sciences Institute (SAMSI) \\ Research Triangle Park, North Carolina, 27709-4006
}

Donald J. Leo

Center for Intelligent Material Systems and Structures

Department of Mechanical Engineering

Virginia Polytechnic Institute and State University

Blacksburg, Virginia, 24061

\begin{abstract}
Application of Rotational Isomeric State (RIS) theory to the prediction of Young's modulus of a solvated ionomer is considered. RIS theory directly addresses polymer chain conformation as it relates to mechanical response trends. Successful adaptation of this methodology to the prediction of elastic moduli would thus provide a powerful tool for guiding ionomer fabrication. The Mark-Curro Monte Carlo methodology is applied to generate a statistically valid number of end-to-end chain lengths via RIS theory for a solvated Nafion case. The distribution of chain lengths is then fitted to a Probability Density Function by the Johnson Bounded distribution method. The fitting parameters, as they relate to the model predictions and physical structure of the polymer, are studied so that a means to extend RIS theory to the reliable prediction of ionomer stiffness may be identified.
\end{abstract}

\section{INTRODUCTION}

Ionic polymers comprise the active layer in Ionic PolymerMetal Composites (IPMCs), which were first identified just over a decade ago [1-5] and now constitute an emerging class of soft transducers (Fig. 1). Because they generate large strain in response to low electric field stimulation and have high gravimetric energy density, there has been considerable conjecture over the potential applications of these soft transducers [2,3,6-8] and considerable fruitful investigations towards these ends [9-11].

The high gravimetric energy density is a function of both the electromechanical transduction mechanisms of the ionic polymer layer and the global IPMC stiffness. Most models of the transduction behavior rely on the cluster morphology of the ionic polymer layer, as proposed by Hsu and Gierke [12], where the material has been solvated with water. In brief, the backbone of the ionic polymer chain is hydrophobic whereas the side chains terminate in hydrophilic ionic groups. Hsu and Geirke propose a clustering of these hydrophilic ionic side groups and the water that has been taken up by the material. The model further suggests an idealized structuring whereby the clusters are of essentially constant radius, uniformly distributed throughout the material, and interconnected by channels. Subsequently, many have sought to quantify the cluster radius and spacing as functions of polymer equivalent weight, hydration level, counterion, etc. 


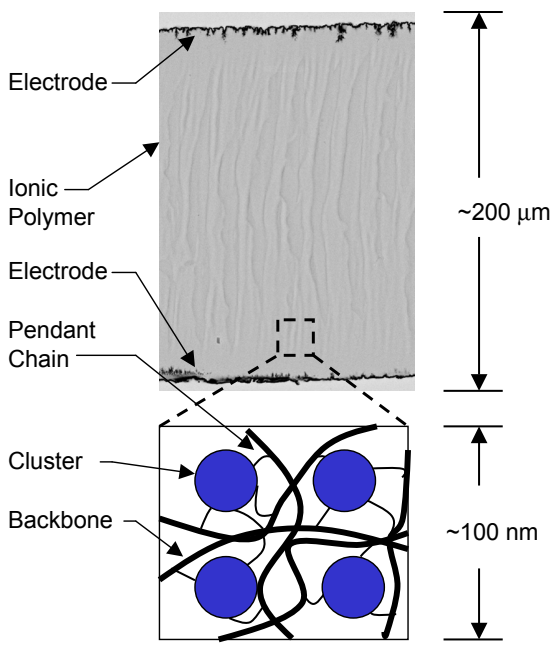

Figure 1. CROSS-SECTIONAL VIEW OF A TYPICAL IPMC WITH ILLUSTRATION OF THE ASSUMED IONIC POLYMER CLUSTERING MORPHOLOGY BASED ON HSU AND GIERKE [12].

- see for instance the modeling works of Datye and coworkers $[13,14]$ and the experimental work of Lehmani et al. [15] and Barbi et al. [16].

Current transduction models tend to be categorized in two classes: models assuming a hydraulic description of the electromechanical response $[17,18]$ and hybrid models including electrostatic, elastic, and osmotic effects $[8,19,20]$. Despite the disagreement over the underlying electromechanical mechanisms, the resulting predictions of all of the above modeling approaches are dependent upon ionomer stiffness. Moreover, it has been noted that the ionomer stiffness affects transducer response both directly, via classic energy density consideration, as well as indirectly, via an impact on transport properties [20,21]. Thus a modeling method which can predict ionomer stiffness as a function of its specific composition (ionomer type, counter ion type, solvation type and level, etc.) is desirable.

Rotational Isomeric State (RIS) theory as described by Flory [23] has long been used as a means to anticipate mechanical response trends in polymeric materials based on the underlying polymer chain conformation. The fundamental idea of this approach is that any given bond within a single polymer chain is limited to a discrete number of possible bond angles, based on corresponding low energy states. From this base, any number of statistical methodologies may be applied to anticipate final chain conformation and corresponding response to load. As discussed by Curro and Mark [24], for the case of short chain polymers, this is best approached with a Monte Carlo methodology, from which a large number of chain end-to-end distances $r$ are generated. The $r$ values can then be used to estimate an appropriate probability density function $P(r)$ for the short chain case, and corresponding material response under load.
The Mark-Curro approach has also been applied to investigations on the effect of particle reinforcement [25-27]. In these particle reinforcement investigations, various volume fractions, inclusion sizes, and inclusion shapes and orientations are considered, where the chain conformation is excluded from occupying any volume dedicated to an inclusion. Thus, the predicted constitutive responses are due solely to the altered conformations of the polymer matrix and do not account for the load carrying capacity of the inclusions. A later preprint [28] suggests that efforts are underway to account for the load carrying capacity of the inclusions as well, and subsequently the methodology may be used for stiffness predictions.

To date this method has not been used for stiffness predictions for reasons largely related to the limitations introduced by way of the simplifying assumptions of RIS theory (i.e., the commonly applied 3-state models of chain bond angles are unable to capture helical chain coiling [29]; uncompensated freeend effects, accurate identification of polymer molecular weight, etc. [22]). However, it has been hypothesized that reasonable estimations for the above noted limitations are possible so long as an appropriate statistical approach is first employed [30,34], where the authors consider various statistical fitting methodologies as they relate to stiffness predictions for solvated ionomers. RIS theory is applied in a manner analogous to the particle inclusion works [25-27] where inclusion volumes are now taken to be clusters. Cluster morphology is assumed a priori, based on available experimental measurements for the various cases; the subsequent backbone conformation in response to this morphology is applied to obtain stiffness predictions. In addition, it is assumed that these clusters, under specified conditions, may act as backbone cross-linking junctions, and thus the ionomer stiffness is based on polymer short chain response. It is found that predictions are sensitive to the choice of statistical methodology, where the Johnson Bounded distribution yields stable predictions as compared to the classically applied Cubic Spline approach. Thus application of this alternate statistical approach represents a tool for expanding the capabilities of this modeling approach to stiffness predictions. In this work, the focus is on understanding how the Johnson Bounded fitting parameters affect stiffness predictions, as well as how they may relate to the physical structure of the ionomer.

The paper is organized as follows. The second section describes the RIS model construction for the ionic polymer Nafion 1100 Equivalent Weight, $\mathrm{Li}^{+}$form, where the assumed cluster morphology is similar to that proposed by Hsu and Gierke [12]. Section three briefly describes the Johnson Bounded fitting approach in contrast with the Cubic Spline approach, and the fourth section presents model predictions and discussion as they relate to the Johnson Bounded fitting parameters. 


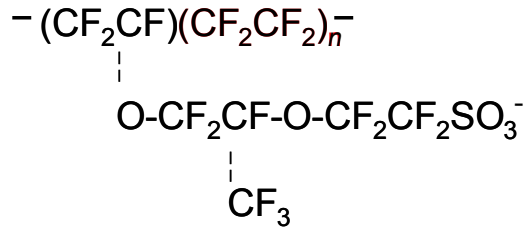

Figure 2. CHEMICAL COMPOSITION OF A NAFION MONOMER.

\section{MULTISCALE MODEL DEVELOPMENT}

The mechanical response of a hydrated Nafion 1100 Equivalent Weight case in salt form is considered, with $\mathrm{Li}^{+}$counterions, where it is assumed that the ion exchange is complete. The MarkCurro approach to RIS theory [24] is applied in a manner analogous to that employed in investigations focused on on the effect of particle reinforcement [25-27]. In the particle reinforcement studies the chain conformation is excluded from occupying any volume dedicated to a rigid inclusion. In this investigation the inclusions take the form of hydrophilic clusters rather than rigid particles. The significant difference in the nature of the inclusion is addressed by way of the additional constraint that the backbone may have discrete points of communication with the hydrophilic clusters via the pendant chain attachment points. These attachment points are assumed to approximate cross-linking points, and thus mark an end-point for the given chain length $r$.

\section{Simulation Model for Estimating Chain Length}

To implement this model, appropriate statistical weight matrices for backbone chain conformation, bond lengths, and distances between pendant chain attachment points must be identified. The repeating monomer unit in the chemical structure of Nafion is depicted in Fig. 2, where $\left(\mathrm{CF}_{2} \mathrm{CF}\right)\left(\mathrm{CF}_{2} \mathrm{CF}_{2}\right)_{n}$ represents the repeating unit of the backbone and $\mathrm{SO}_{3}^{-}$is the pendant chain terminal ionic group which tends toward the hydrophilic clusters. If the $87 / 13$ Nafion case is considered, meaning that there are approximately $13\left(\mathrm{CF}_{2} \mathrm{CF}\right)$ groups to every 87 $\left(\mathrm{CF}_{2} \mathrm{CF}_{2}\right)$ groups in the total length of the backbone, then $n$ is most often approximately 7 . In other words, the bulk of the backbone takes the form of PTFE, or Teflon [31,32]. Because it is the backbone of the polymer which is responsible for the load carrying capacity of the ionomer, the PTFE conformation is assumed for all bond placements except pendant chain attachment points.

Whereas a typical value for $n$ in the chemical structure of Nafion is approximately 7 , there is in fact variability in this value. To account for this variation, $n$ is sampled from a discrete probability distribution with a minimum of 5 , a maximum of 11 , and a mean of approximately 7 [30].

Still to be addressed is the number of times the repeat unit of Fig. 2 is expected for a single polymer strand of Nafion. Because the actual molecular weight is not readily available, in part because polymers do not have fixed chain lengths, a distribution of lengths is assumed. The assumed distribution anticipates that to-

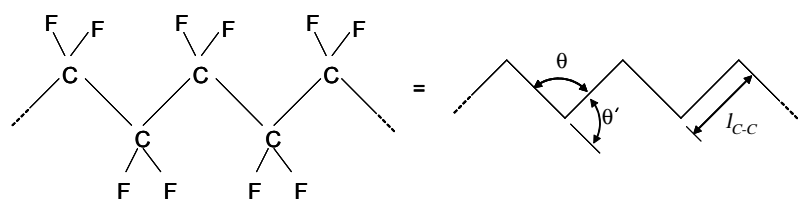

Figure 3. DEPICTION OF THE IN-PLANE BOND ANGLE $\theta=116^{\circ}$ AND PTFE BACKBONE COMPONENT LENGTH $l_{\mathrm{C}-\mathrm{C}}=1.53 \AA$ A. THE ANGLE $\theta^{\prime}$ IS THE SUPPLEMENTARY ANGLE TO $\theta$ HAVING THE VALUE $64^{\circ}$.

tal chain lengths contain between 135 and 225 repeat units. Note, however, that the lack of available information on this parameter is considered non-critical because this material's load bearing capability is based on the distance between cluster communication points along a given backbone rather than on the entire backbone chain length.

The model progression begins with the placement of a cluster configuration in a 3 -dimensional grid of $(5000 \AA)^{3}$. The grid size is selected to accommodate the longest anticipated, fully extended backbone. Two cluster distribution schemes have been considered as detailed in the preliminary works [30,34]. The first relies directly on the simplified model of Hsu and Gierke [12] where the clusters are uniformly distributed on a square grid analogous to the cubic crystalline structure. The second uniformly distributes clusters of the same radius and net volume fraction as the first case, but on a staggered grid analogous to the HCP crystalline structure. As noted in [34], it is found that both distributions yield similar predicted stress-strain trends, with the HCP distribution leading to overall stiffer predicted response. The cluster size and volume fraction for the cases studied are based on [8]. In the current paper, only the Cubic cluster distribution is considered.

Once the cluster distribution is assigned, a single PTFE bond is randomly placed in the 3-dimensional grid, where cluster locations are excluded. For PTFE the in-plane bond angle and length are fixed at $\theta=116^{\circ}$, and $l_{\mathrm{C}-\mathrm{C}}=1.53 \AA$, respectively, as illustrated in Fig. 3. Thus, the in-plane angle of the second bond is known; however, a statistical weight matrix must be applied in order to identify an appropriate out-of-plane rotation angle, where it is understood that various angular orientations between bonds correspond with local low energy conformations. Each subsequent bond must be similarly placed. The 4-state statistical weight matrices for PTFE are applied [29]. For the 4-state case the possible out-of-plane angles are $+15^{\circ},+120^{\circ},-120^{\circ}$, and $-15^{\circ}$ (or trans+, gauche+, gauche-,trans-).

The 4-state matrices are applied to all bond placements with two exceptions. The matrices are overridden when a bond placement coincides with a cluster or when the bond corresponding to a pendant chain attachment point is being placed. The first exception addresses the reality that the backbone chain is hydrophobic and will reconfigure upon solvation to an alternate low energy state in order to avoid the hydrophilic region. The second ex- 


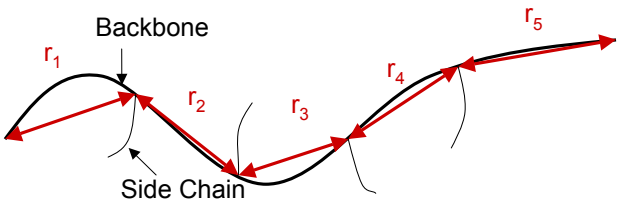

Figure 4. DISTANCE $r$ BETWEEN CLUSTER INTERACTION POINTS FOR A SINGLE NAFION POLYMER CHAIN.

ception addresses the reality that the terminal ionic group will tend toward a hydrophilic region and again locally reconfigure the chain upon solvation. It is understood that application of the in-plane PTFE bond angle in this case is a simplifying assumption.

When a bond placement would locate the next bond within a cluster, another random number is generated to find an alternate but viable low energy conformation. If all out-of-plane rotation angles result in cluster coincidence, the chain is terminated. When a bond corresponding to a pendant chain connection point is being placed, of the possible $\pm 15^{\circ}$ and $\pm 120^{\circ}$ out-of-plane PTFE rotations, the angle that minimizes the distance between the carbon atom with the attached side chain and the nearest cluster is used. It is assumed that the pendant chain terminal ionic group successfully communicates with the nearest cluster if the distance between the backbone connection point and the nearest cluster is within $8 \AA$. This value is an estimate of the fully extended length of the pendant chain. It is found that application of this value leads to $83 \%$ successful cluster communication for the cluster configuration considered here. It is not expected that $100 \%$ of the ionic groups will reside within a cluster and thus, this rate is deemed reasonable.

Each simulated Nafion backbone chain can have multiple $r$ values as illustrated in Fig. 4. Furthermore, the first and last $r$ value for each chain, or free ends, do not support any load, and therefore do not contribute to stiffness. Correction for the freeend effects are addressed by discarding the first and last $r$ value of each simulated chain. In order to assure statistical validity, a large number of $r$ values (about 10,000) are generated.

\section{Macroscopic Model for Estimating Stiffness}

Assuming an appropriate $P(r)$ expression has been developed, per Boltzmann's approach to statistical thermodynamics, it can be directly related to the entropy of the chain as a function of the number of conformations available for a given state [24],

$$
S(r)=c+k \ln P(r)
$$

where $k$ is Boltzmann's constant and $c$ is a constant of integration which drops out when the difference in entropy for the unperturbed configuration is taken with respect to the distorted config- uration. In this approach it is assumed that under load the rotation about the individual bonds is unrestricted which allows the assumption that the Helmholz free energy is strictly a function of entropy. For the assumptions of rubberlike elasticity, the "three chain" model, as described by Treloar [31], yields the relation

$$
\Delta S=\frac{v}{3}\left[S\left(r_{\mathrm{o}} \alpha\right)+2 S\left(r_{\mathrm{o}} \alpha^{-1 / 2}\right)-3 S\left(r_{\mathrm{o}}\right)\right]
$$

for the change in entropy upon distortion. Here $v$ is the number density of network chains, $r_{\mathrm{o}}$ is the root mean square of the $r$ values applied in the development of $P(r)$, and $\alpha=L / L_{i}$ is the relative length of the sample. The nominal stress $f^{*}$ is then given by

$$
\begin{aligned}
f^{*} & =-T\left(\frac{\partial \Delta S}{\partial \alpha}\right)_{T} \\
& =-\frac{v k T r_{\mathrm{o}}}{3}\left[G^{\prime}\left(r_{\mathrm{o}} \alpha\right)-\alpha^{-3 / 2} G^{\prime}\left(r_{\mathrm{o}} \alpha^{-1 / 2}\right)\right]
\end{aligned}
$$

where $G(r)=\ln P(r)$ and $G^{\prime}(r)=\frac{d G(r)}{d r}$. The corresponding modulus $\left[f^{*}\right]$ is given as

$$
\left[f^{*}\right]=\frac{f^{*}}{\alpha-\alpha^{-2}} .
$$

For small strains $(\alpha \rightarrow 1)$, the modulus in (4) approaches Young's modulus for the polymer matrix alone.

\section{STATISTICAL ANALYSIS}

The details of the statistical fitting methods applied to the development of various $P(r)$ expressions for the same distribution of $r$ values are discussed in [30]. Provided here is a brief overview of the cubic spline and Johnson Bounded methods. In the traditional approach of RIS Monte Carlo theory, a cubic spline approach is used to estimate $P(r)$. The simulationgenerated $r$ values are divided into groups, or bins, and a knot value is specified for each bin so that on each interval (delimited by the knots), a cubic polynomial is fit to the data. If we define the indicator function $L_{i}(r)$ for the subinterval $\left(k_{i}, k_{i+1}\right]$, where $k_{i}$ is the $i$ th knot, as

$$
L_{i}(r)=\left\{\begin{array}{l}
1, \text { for } k_{i}<r \leq k_{i+1} \\
0, \text { otherwise }
\end{array}\right.
$$


then the result is a piecewise-polynomial estimate of $P(r)$ of the form

$$
\begin{aligned}
P(r)= & \frac{1}{K} \sum_{i=1}^{n^{*}} L_{i}(r)\left[A_{i}\left(r-k_{i}\right)^{3}+B_{i}\left(r-k_{i}\right)^{2}\right. \\
& \left.+C_{i}\left(r-k_{i}\right)+D_{i}\right]
\end{aligned}
$$

where $n^{*}$ is the number of knots, $A, B, C$, and $D$ are the corresponding coefficients of the cubic equations, and $K$ is a normalizing constant so that the function $P(r)$ given in (6) integrates to unity. While this expression is easily differentiable for substitution into equations (1)-(4), one challenge with this approach is that cubic polynomials are often not sufficiently flexible to accurately model the tail behavior of the density. Additionally, the shape of the estimated density is highly dependent on the number and location of the knots. For example, if the number of knots is large, the corresponding estimate of $P(r)$ not only gives the visual impression of being unnaturally noisy, but also yields nonphysical response trends. Finally, the details of the fitting methodology (such as knot selection) are typically not welldefined.

Alternatively, the application of the Johnson family of distributions for density estimation is clearly defined, effectively eliminating any ambiguity in defining the fitting procedure. Further, unlike the coefficients in the cubic spline approach, the various parameters of the Johnson distribution have specific statistical meaning. Lastly, the Johnson distribution only requires the estimation of four parameters and the resulting expression can be easily applied in equations (1)-(4).

In general, the probability density function $P(r)$ based on the Johnson distributions has the form

$$
P(r)=\frac{\delta}{\lambda \sqrt{2 \pi}} f^{\prime}\left(\frac{r-\xi}{\lambda}\right) \exp \left\{-\frac{1}{2}\left[\gamma+\delta f\left(\frac{r-\xi}{\lambda}\right)\right]^{2}\right\},
$$

where $\delta$ and $\gamma$ are shape parameters, $\lambda$ is a scale parameter, $\xi$ is a location parameter, the function $f(\cdot)$ is defined according to the applied distribution family, and $f^{\prime}(\cdot)$ is the first derivative of $f(\cdot)$ with respect to $r[30,33]$. The distribution families defining $f(\cdot)$ are

$$
f(y)= \begin{cases}\ln (y), & \text { for the } S_{L} \text { (lognormal) family, } \\ \ln \left(y+\sqrt{y^{2}+1}\right), & \text { for the } S_{U} \text { (unbounded) family } \\ \ln \left(\frac{y}{1-y}\right), & \text { for the } S_{B} \text { (bounded) family } \\ y, & \text { for the } S_{N} \text { (normal) family; }\end{cases}
$$

The bounds on these distributions are

$$
\mathbf{H}= \begin{cases}{[\xi,+\infty)} & \text { for the } S_{L} \text { (lognormal) family } \\ (-\infty,+\infty) & \text { for the } S_{U} \text { (unbounded) family } \\ {[\xi, \xi+\lambda]} & \text { for the } S_{B} \text { (bounded) family } \\ (-\infty,+\infty) & \text { for the } S_{N} \text { (normal) family }\end{cases}
$$

Using equations (7)-(9), the function $G(r)$ can be written as

$$
G^{\prime}(r)=\frac{f^{\prime \prime}\left(\frac{r-\xi}{\lambda}\right)-\delta\left[f^{\prime}\left(\frac{r-\xi}{\lambda}\right)\right]^{2}\left[\gamma+\delta f\left(\frac{r-\xi}{\lambda}\right)\right]}{\lambda f^{\prime}\left(\frac{r-\xi}{\lambda}\right)} .
$$

When this expression is substituted into equation (4), the resulting stiffness expression as a function of distortion is given by

$$
\begin{aligned}
& {\left[f^{*}\right]=-\frac{r_{\mathrm{o}} \nu k T}{3\left(\alpha-\alpha^{-2}\right)} \cdot} \\
& \left\{\frac{f^{\prime \prime}\left(\frac{r_{0} \alpha-\xi}{\lambda}\right)-\delta\left[f^{\prime}\left(\frac{r_{0} \alpha-\xi}{\lambda}\right)\right]^{2}\left[\gamma+\delta f\left(\frac{r_{0} \alpha-\xi}{\lambda}\right)\right]}{\lambda f^{\prime}\left(\frac{r_{0} \alpha-\xi}{\lambda}\right)}-\right. \\
& \left.\frac{f^{\prime \prime}\left(\frac{r_{0} \alpha^{-1 / 2}-\xi}{\lambda}\right)-\delta\left[f^{\prime}\left(\frac{r_{0} \alpha^{-1 / 2}-\xi}{\lambda}\right)\right]^{2}\left[\gamma+\delta f\left(\frac{r_{0} \alpha^{-1 / 2}-\xi}{\lambda}\right)\right]}{\alpha^{3 / 2} \lambda f^{\prime}\left(\frac{r_{0} \alpha^{-1 / 2}-\xi}{\lambda}\right)}\right\} .
\end{aligned}
$$

Of the four Johnson family distributions initially considered (unbounded, bounded, lognormal, and normal), it is found that the lognormal and normal families are not sufficiently flexible for the data generated from the simulation model and both yield poor statistical fits, while the unbounded family yields significant scatter in subsequent stiffness predictions. Subsequently, only the bounded family is considered in detail.

\section{PREDICTIONS AND DISCUSSION}

While a total of 135 to 225 repeat units (Fig. 2) per ionomer chain is identified as the target, the chain length is typically terminated much earlier due to chain collision with a cluster. Actual simulated chain lengths $m$ never exceeded 45 . While early termination clearly leads to non-physical predictions of total chain length, it is not believed that this early termination presents a significant problem in the development of an appropriate $P(r)$ expression because it is the lengths between cluster interactions which dictate the elastic properties of the material. Furthermore, correction of free-end effects not only addresses the reality that 
free ends do not contribute to material stiffness, but also necessarily eliminates early termination effects. (For discussion of free end effects, see for instance, Treloar [22].)

Perturbation studies yield insight into the physical significance of the various Johnson parameters. Table 1 and Figures 5-7 illustrate the variations observed in the predicted probability density functions and stiffness as the Johnson bounded coefficients are varied; the location of the root mean square of the $r$-values used to develop $\mathrm{P}(r), r_{\mathrm{o}}$, is indicated on each curve.

Table 1. PERTURBATION STUDY OF JOHNSON BOUNDED PARAMETERS (CUBIC CLUSTER DISTRIBUTION ONLY).

\begin{tabular}{cccccc}
\hline \hline$\xi$ & $\delta$ & $\lambda$ & $\gamma$ & $r_{\mathrm{o}}(\AA)$ & $E_{i}(\mathrm{MPa})$ \\
\hline-0.72 & 3.096 & 527.0 & 10.87 & 16.14 & 9.0 \\
3.28 & 3.096 & 527.0 & 10.87 & 19.97 & 13.6 \\
7.28 & 3.096 & 527.0 & 10.87 & 23.86 & 19.1 \\
\hline 3.28 & 2.896 & 527.0 & 10.87 & 16.63 & 12.5 \\
3.28 & 3.096 & 527.0 & 10.87 & 19.97 & 13.6 \\
3.28 & 3.296 & 527.0 & 10.87 & 23.58 & 14.8 \\
\hline 3.28 & 3.096 & 523.0 & 10.87 & 19.84 & 13.5 \\
3.28 & 3.096 & 527.0 & 10.87 & 19.97 & 13.6 \\
3.28 & 3.096 & 531.0 & 10.87 & 20.10 & 13.6 \\
\hline 3.28 & 3.096 & 527.0 & 9.87 & 26.06 & 12.8 \\
3.28 & 3.096 & 527.0 & 10.87 & 19.97 & 13.6 \\
3.28 & 3.096 & 527.0 & 11.87 & 15.46 & 14.9 \\
\hline
\end{tabular}

As reported in [34], it is found that, in general, variation of $\xi$ (location parameter) has the most significant impact on predicted stiffness; variation of $\delta$ (shape/peak parameter) and $\lambda$ (scale/spread parameter) have weaker but discernable effects; and variation of $\gamma$ (shape/skewness parameter) has a variable effect.

While the ultimate goal of this modeling approach is reliable stiffness predictions, such as Young's modulus, in the current model only normalized stiffness predictions are considered. Issues that must be addressed before the model is expected to yield accurate stiffness predictions include accounting for the semi-crystalline nature of the polymer matrix, which is currently neglected, and further study of potential effects of early termination. Figure 8 illustrates a sample stress-strain plot where the stiffness has been normalized with respect to $v k T$. As noted above, increasing $\xi$ leads to a predicted increase in stiffness. The normalized plots for variation of $\delta$ and $\gamma$ are directly analogous,

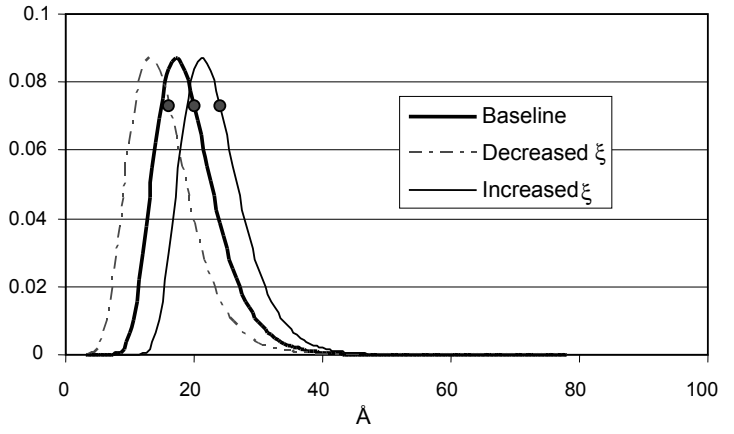

Figure 5. PDF CURVES FOR THE DISTRIBUTION OF $r$-VALUES AND CORRESPONDING ROOT MEAN SQUARE VALUE, $r_{0}$, FOR VARIATIONS IN THE $\xi$ JOHNSON BOUNDED COEFFICIENT.

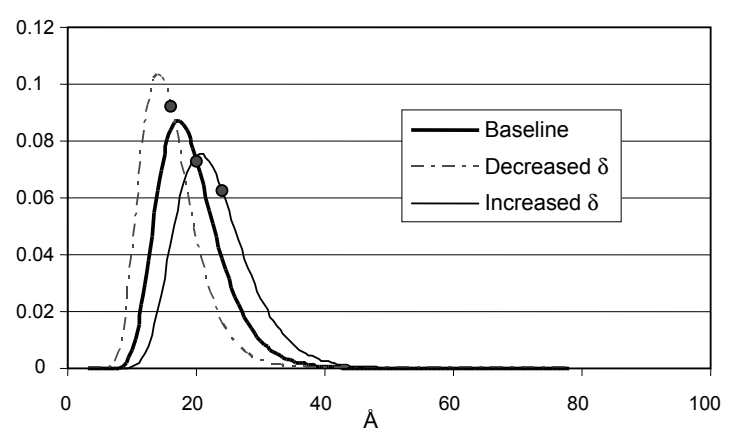

Figure 6. PDF CURVES FOR THE DISTRIBUTION OF $r$-VALUES AND CORRESPONDING ROOT MEAN SQUARE VALUE, $r_{\mathrm{o}}$, FOR VARIATIONS IN THE $\delta$ JOHNSON BOUNDED COEFFICIENT.

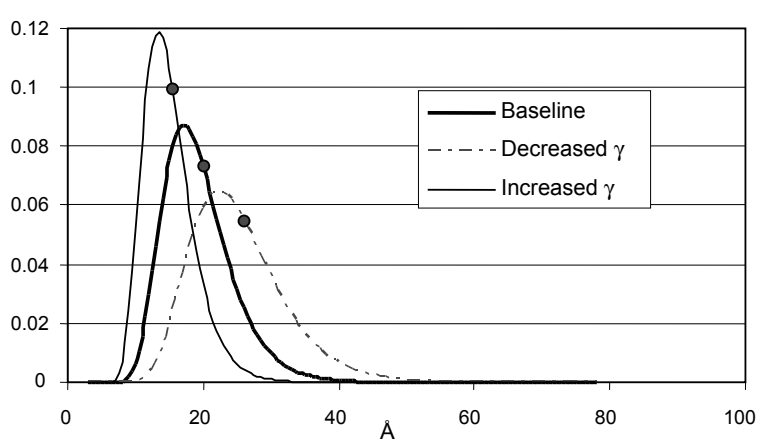

Figure 7. PDF CURVES FOR THE DISTRIBUTION OF $r$-VALUES AND CORRESPONDING ROOT MEAN SQUARE VALUE, $r_{0}$, FOR VARIATIONS IN THE $\gamma$ JOHNSON BOUNDED COEFFICIENT. 


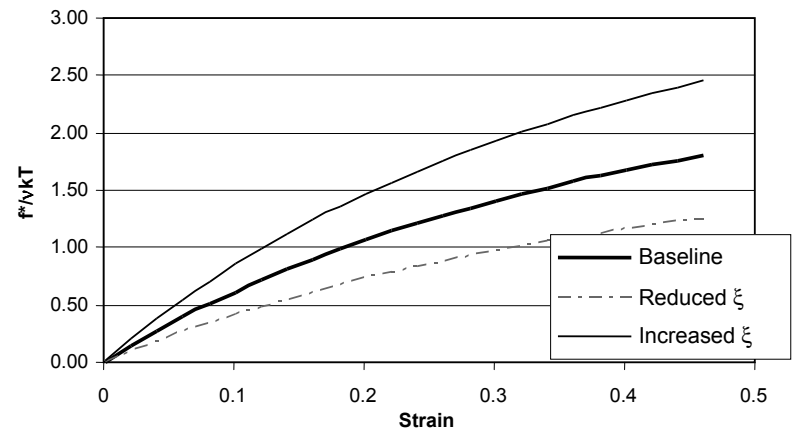

Figure 8. STRESS NORMALIZED WITH RESPECT TO $v k T$ VERSUS STRAIN FOR VARIATIONS IN THE $\xi$ JOHNSON BOUNDED COEFFICIENT.

where increases in each also lead to predicted increases in stiffness.

Increasing $\xi$, as illustrated in Figures 5 and 8, always leads to an increase in the predicted stiffness. To interpret the physical significance of this, the analysis must be considered within the context of Equation (11) and the definition of chain density,

$$
v=\frac{\rho N_{\mathrm{A}}}{\mathrm{MW}_{\mathrm{c}-\mathrm{c}}}
$$

where $\rho$ is the material density, $N_{\mathrm{A}}$ is Avagadro's number, and $\mathrm{MW}_{\mathrm{c}-\mathrm{c}}$ is the molecular weight of the portion of the chain between two cluster communication points. From these equations it is seen that the chain density $v$ is held constant while effectively increasing the typical chain length. In order for this to happen both the material density $\rho$ and molecular weight must rise, meaning that any given bond within the higher $\xi$ material is carrying less load for a given external load. This corresponds to an increase in the load carrying capability for the bulk material, or higher stiffness.

Increasing $\delta$ or decreasing $\lambda$ have similar effects, on the distribution, where both lead to a more peaked distribution and marginally higher predicted stiffness; variation of $\delta$ is illustrated by Figure 6. To say that a distribution is more peaked also suggests that the molecular weight distribution is narrower. It has been found that a narrower molecular weight distribution corresponds to a stiffer material in other polymers such as HDPE, so it is within reason that the same would be true for this class of polymers.

The effect of varying $\gamma$ on the predicted PDF is illustrated by Figure 7 . While variation of this parameter does effect the predicted stiffness, the nature of this effect is not consistent [34]. Because this skewness parameter effectively addresses variations in the relative proportions of long and short $r$ values, this per- ceived lack of consistency is likely based on the relative skewness of the baseline case.

It is worthy of mention that the traditional cubic spline approach is not studied in detail because it is deemed the most unreliable of the considered methods due largely to the ill-defined methodology for fixing the knots. Further, as discussed in the previous works [30,34], minor variation to the number and placement of knots in the cubic spline approach has non-negligible impact on predicted stiffness. The single greatest challenge with cubic spline is discerning the level of detail which is physically appropriate. With the Johnson cases, no qualitative judgment of this sort is required.

Moreover, the stable nature of the stiffness predictions for the Johnson bounded case is mirrored in stable stress-strain trends as illustrated above, while the cubic spline case is much more likely to have erratic stress-strain response (mirroring the erratic stiffness predictions). It is thus again emphasized that application of the Johnson bounded case represents both stability and simplification in RIS predictions as compared to the cubic spline case.

\section{CONCLUSIONS}

The development of a new multiscale modeling technique for the prediction of ionomer stiffness has been considered through extension of traditional RIS theory. Prediction stability is introduced by fitting the chain length distribution $P(r)$ using a Johnson bounded distribution rather than the commonly applied cubic spline approach. A Johnson bounded distribution is preferred for its stability and because its parameters can be related to physical characteristics in the polymer, whereas cubic spline parameters cannot. Variation of the Johnson bounded parameter $\xi$ has the most significant effect on stiffness predictions, where increasing $\xi$ leads to increased stiffness prediction; variation of this parameter is related directly to polymer chain conformation and the subsequent load carrying capacity of the material. Similar assessments may be made for the Johnson bounded parameters $\delta, \lambda$, and $\gamma$, but where the effect on the predicted stiffness is less pronounced. Direct prediction of stiffness and stress-strain response may ultimately be possible once sources of error such as the effect of polymer semi-crystallinity and early termination are addressed in detail.

\section{ACKNOWLEDGMENT}

The authors would like to thank Garth Wilkes (Virginia Tech) for multiple enlightening discussions on RIS theory and Jim Wilson (NCSU) for input regarding the density fitting techniques . Collaboration was facilitated while the authors were visitors at the Statistical and Applied Mathematical Sciences Institute (SAMSI), Research Triangle Park, NC, and the research of EKL was supported in part by the Science Foundation through 
the SAMSI grant DMS-0112069. LMW and DJL were supported by National Science Foundation Grant CMS 0093889 whereas RCS was supported in part by the Air Force Office of Scientific Research through the grant AFOSR FA9550-04-1-0203. The authors gratefully acknowledge this support.

\section{REFERENCES}

[1] K. Sadeghipour, R. Salomon, and S. Neogi: Development of a novel electrochemically active membrane and 'smart' material based vibration sensor/damper. Smart Mater. Struc. 1(2), 172 (1992).

[2] K. Oguro, Y. Kawami, and H. Takenaka: An actuator element of polyelectrolyte gel membrane-electrode composite. Osaka Kogyo Gijutsu Shikensho Kiho 43(1), 21 (1992).

[3] Yoshito Osada and Mariko Hasebe: Electrically Activated Mechanochemical Devices Using Polyelectrolyte Gels. Chem. Lett. 1285 (1985).

[4] Masahiro Irie: Photoresponsive Polymers. Reversible Bending of Rod-Shaped Acrylamide Gels in an Electric Field. Macromolecules 19, 2890 (1986).

[5] Tohru Shiga and Toshio Kurauchi: Deformation of Polyelectrolyte Gels Under the Influence of Electric Filed. Journal of Applied Polymer Science 39(11/12), 2305 (1990).

[6] M. Shahinpoor: Conceptual Design, Kinematics and Dynamics of Swimming Robotic Structures Using Ionic Polymeric Gel Muscles. Smart Materials and Structures 1(1), 91 (1992).

[7] M. Shahinpoor, Y Bar-Cohen, J.O. Simpson, and J. Smith: Ionic polymer-metal composites (IPMCs) as biomimetic sensors, actuators and artificial muscles - a review. Smart Mater. Struct. 7(6), R15 (1998).

[8] Y. Bar-Cohen (Editor), Electroactive Polymer (EAP) Actuators as Artificial Muscles - Reality, Potential, and Challenges (SPIE- The International Society for Optical Engineering, Bellingham, Washington, 2001).

[9] M. Mojarrad and M. Shahinpoor: Ion exchange membraneplatinum composites as electrically controllable artificial muscles. SPIE Proc 2779 p. 1012 (1996).

[10] S. Nemat-Nasser: Micromechanics of Actuation of Ionic Polymer-metal Composites (IPMCs). J. Appl. Phys. 92(5), 2899 (2002).

[11] K. Farinholt, K. Newbury, M. Bennet, and D. Leo: An Investigation into the Relationship between Charge and Strain in Ionic Polymer Sensors, in First World Congress on Biomimetics and Artificial Muscles (Albuquerque, NM, 9-11 December 2002).

[12] W.Y. Hsu and T.D. Gierke: Elastic Theory for Ionic Clustering in Perfluorinated ionomers. Macromolecules 15, 101 (1982).

[13] V. K. Datye, P.L. Taylor, and A. J. Hopfinger: Simple
Model for Clustering and Ionic Transport in Ionomer Membranes. Macromolecules 17, 1704 (1984).

[14] V. K. Datye and P. L. Taylor: Electrostatic Contributions to the Free Energy of Clustering of an Ionomer. Macromolecules 18, 1479 (1985).

[15] A. Lehmani, S. Durand-Vidal, and P. Turq: Surface Morphology of Nafion 117 Membrane by Tapping Mode Atomic Force Microscope. J. Appl. Poly. Sci. 68, 503 (1998).

[16] V. Barbi, S. Funari, R. Gehrke, N. Scharnagl, and N. Stribeck: Nanostructure of Nafion membrane material as a function of mechanical load studied by SAXS. Polymer 44, 4853 (2003).

[17] P.G. de Gennes, Ko Okumura, M. Shahinpoor, and K.J. Kim: Mechanoelectric Effects in Ionic Gels. Europhysics Letters 50(4), 513 (2000).

[18] K. Asaka and K. Oguro: Bending of polyelectrolyte membrane platinum composites by electric stimuli. J. Elec. Chem. 480, 186 (2000).

[19] J. Y. Li and S. Nemat Nasser: Micromechanical analysis of ionic clustering in Nafion perfluorinated membrane. Mech. Mater. 32, 303 (2000).

[20] S. Nemat-Nasser and J.Y. Li: Electromechanical response of ionic polymer-metal composites. J. Appl. Phys. 87(7), 3321 (2000).

[21] L.M. Weiland and D.J. Leo: Computational Analysis of Ionic Polymer Cluster Energetics. J. Appl. Phys. 97, 013541 (2004).

[22] L.R.G. Treloar, The Physics of Rubber Elasticity, 3rd ed. (Clarendon Press, Oxford, England, 1975).

[23] P.J. Flory, Statistical Mechanics of Chain Molecules (Hanser Publishers, New York, New York, 1988).

[24] J.E. Mark and J.G. Curro: A non-Gaussian theory of rubberlike elasticity based on rotational iomeric state simulations of network chain configurations. I. Polyethylene and Polydimethylsiloxane short-chain unimodal networks. J. Chem. Phys. 79(11), 5705 (1983).

[25] Q.W. Yuan, A. Kloczkowski, J.E. Mark, and M.A. Sharaf: Simulations on the Reinforcement of Poly(dimethylsiloxane) Elastomers by Randomly Distributed Filler Particles. J. Poly. Sci B: Poly. Phys 34, 1647 (1996).

[26] M.A. Sharaf, A.Kloczkowski, and J.E. Mark: Monte Carlo simulations on reinforcement of an elastomer by oriented prolate particles. Comp. Theo. Poly. Sci. 11, 251 (2001).

[27] M.A. Sharaf, R.H. Abouhussein: Computer Simulations on the chain deformations of Poly(Ethylene) by RandomlyOriented Prolate Filler particles Arranged on a Cubic Lattice. Poly. Preprints 44(1), 1258 (2003).

[28] M.A. Sharaf, I.M. Jasiuk, K.I. Jacob: Monte Carlo Simulations combined with Micromechanics to predict effective elastic moduli of elastomeric nanocomposites filled with unidirectional rigid inclusions. Poly. Preprints 44(1), 1249 (2003).

[29] T.W. Bates and W.H. Stockmayer: Conformational Ener- 
gies of Perflouroalkanes. II Dipole Moments of H(CF2)nH. Macromolecules 1(1), 12 (1968).

[30] J.L. Mathews, E.K. Lada, L.M. Weiland, R.C. Smith, D.J. Leo: Monte Carlo Simulation of a Solvated Ionic Polymer with Cluster Morphology. Submitted to Smart Materials and Structures January 2005.

[31] K.A. Page and R.B. Moore: Correlations between bulk mechanical relaxations and spin diffusion times in Perfluorosulfonate Ionomers: Molecular Origins of Mechanical Relaxations. Polymer Preprints 44(1), 1144 (2003).

[32] A.K. Phillips and R.B. Moore: Phase Behavior in Solution Processed Perfluorosulfonate Ionomers. Polymer Preprints 44(1), 1142 (2003).

[33] D.J. DeBrota, R.S. Dittus, S.D. Roberts, J.R. Wilson, J.J. Swain, and S. Venkatraman: Modeling Input Processes with Johnson Distributions. Proc. of the Winter Simulation Conf. p. 308 (Piscataway, New Jersey: IEEE, 1989).

[34] L.M. Weiland, E.K. Lada, R.C. Smith, and D.J. Leo: Application of Rotational Isomeric State Theory to Ionic Polymer Stiffness Predictions. J. Mat. Sci., accepted May 2005. 